\title{
Cost-Effectiveness of Peer-Educator-Delivered Lifestyle Modification for Type 2 Diabetes Prevention in a Young Healthy Population in Sri Lanka: A Trial-Based Economic Evaluation and Economic Model
}

\author{
James Shearer ${ }^{1} \mathbb{D} \cdot$ Miral Kalyani $^{2} \cdot$ Anastasios Mangelis $^{3} \cdot$ Dileep de Silva $^{4} \cdot$ Padmal de Silva $^{5} \cdot$ Mahen Wijesuriya $^{6}$. \\ Janaka Karalliedde ${ }^{3}$
}

Accepted: 17 June 2021 / Published online: 12 July 2021

(c) The Author(s) 2021

\begin{abstract}
Background This study evaluated the cost effectiveness of an intensive lifestyle modification (LSM) intervention delivered by peer educators for the prevention of type 2 diabetes mellitus in a young at-risk population in a low healthcare resource setting. Objective The aim of this study was to evaluate the short-term and long-term cost effectiveness of an intensive lifestyle modification intervention for type 2 diabetes prevention in a young urban at-risk population in Sri Lanka.

Methods This was an economic evaluation using cost and outcome data from a randomized controlled trial. We randomized 3539 healthy individuals aged 5-40 years with risk factors for type 2 diabetes to either 3-monthly (P-LSM $n=1727)$ or 12-monthly (C-LSM $n=1812$ ) peer-educator advice aimed at reducing weight, improving diet, reducing psychological stress and increasing physical activity. A cost-effectiveness analysis was conducted from a health system perspective with outcomes expressed as disability-adjusted life-years (DALYs). Intervention costs and outcomes were collected during a median clinical trial period of 3 years and extrapolated to a lifetime horizon using economic modelling. Uncertainty in the lifetime model was explored by structural and probabilistic sensitivity analyses.

Results The costs of the more intensive peer support programme were partially offset by reduced costs of type 2 diabetes complications recorded over the trial period and completely offset by lifetime cost savings of 6000 LKR. The more intensive P-LSM also averted more DALYs, estimated at 0.456 DALYs over the lifetime of participants.

Conclusions In a young at-risk Sri Lanka population, an intensive LSM programme was cost effective, averting more DALYs at an acceptable additional cost than a much less intensive LSM programme. Early intervention in young at-risk people represents good value for money from the Sri Lankan health care payer perspective.
\end{abstract}

James Shearer

James.shearer@kcl.ac.uk

1 King's Health Economics, Institute of Psychiatry, The David Goldberg Centre, Psychology and Neuroscience at King's College London, De Crespigny Park, Box P024, London SE5 8AF, UK

2 London School of Hygiene and Tropical Medicine, London, UK

3 School of Cardiovascular Medicine and Sciences, King's College London, London, UK

4 Health Human Resources, Ministry of Health, Colombo, Sri Lanka

5 National Institute of Health Sciences, Kalutara, Sri Lanka

6 National Diabetes Centre, Rajagiriya, Sri Lanka

\section{Key Points for Decision Makers}

Efforts to reduce the global health and economic burden of diabetes mellitus should emphasize prevention of diabetes or delaying its onset, through enhancing health behaviours and diets at the population level, and early detection and management of high-risk individuals.

This economic evaluation indicates that lifestyle interventions delivered at low intensity may minimize budget impact in the short term, but more intensive lifestyle modification interventions delivered at younger ages will maximize reductions in diabetes incidence and costs in the long term and are therefore better value for money. 


\section{Introduction}

The growing burden of type 2 diabetes mellitus has emerged as a major public health challenge in low- and middle-income countries (LMICs). Currently, around $80 \%$ of people with diabetes live in LMICs and it is estimated that the largest increases in prevalence will take place in regions transitioning from low to middle income levels [1]. Asia is at the epicentre of this growing epidemic, accounting for over $60 \%$ of type 2 diabetes cases worldwide [2, $3]$. While traditionally considered a disease affecting older age groups; the prevalence of type 2 diabetes has become increasingly common in children, adolescents, and young adults [4-6]. In South Asia, almost a third of future cases are predicted to occur in those aged $<45$ years [6].

Type 2 diabetes can lead to a number of disabling and fatal complications such as cardiovascular disease, chronic kidney disease, neuropathy and vision loss [1]. Management of type 2 diabetes and its associated complications is complex, time consuming and costly. A recent cost-ofillness study estimated the global annual cost of diabetes for 2015 at US\$1.31 trillion [7]. This economic burden is borne disproportionately by LMICs where a large proportion of health spending is in the form of out-of-pocket payments [8].

Several large-scale trials have demonstrated the effectiveness of lifestyle modification (LSM) interventions in preventing or delaying the onset of type 2 diabetes among high-risk individuals [9-13]. Furthermore, lifestyle modification interventions have been demonstrated to be cost effective and even cost saving in various, predominantly high-income contexts [14-21]. Evidence of effectiveness and cost effectiveness of LSM interventions in low- and middle-income countries, however, is scarce.

Recent research has demonstrated the efficacy of LSM as an early intervention for type 2 diabetes prevention in a young at-risk population in Sri Lanka [6]. The Prevention of Cardio-Metabolic Disease with Lifestyle Modification in Sri Lanka (DIABRISK-SL) study screened young urban Sri Lankans within the Colombo District aged 5-40 years for four risk factors-physical inactivity, raised waist circumference, raised body mass index (BMI) and firstdegree family history of type 2 diabetes. Participants with two or more risk factors were randomized into an intensive peer-educator-delivered tri-monthly LSM intervention (P-LSM) or a less intensive annual control LSM intervention (C-LSM). Following median follow up of 3 years, the study found significant reduction in the incidence of new-onset type 2 diabetes, impaired fasting glucose and impaired glucose tolerance [6].

The aim of this study was to evaluate the cost effectiveness of an intensive lifestyle modification intervention for type 2 diabetes prevention in a young urban at-risk population in Sri Lanka. In order to capture the longer-term impact of lifestyle modification on disease progression and costs, we modelled cost, disease progression and outcome data from the DIABRISK-SL trial and existing literature.

\section{Methods}

\subsection{Clinical Trial}

The DIABRISK-SL study was an open, randomized, controlled, parallel-group clinical trial of early LSM intervention in a young population at risk for type 2 diabetes in Colombo, the largest urban district in Sri Lanka. The study screened and recruited participants between the ages of 5-40 years from workplaces, schools, community organizations and universities. Participants with two or more risk factors for cardio-metabolic disease were invited to participate in the trial. A total of 3539 participants were recruited and randomized to P-LSM $(n=1726)$ or C-LSM $(n=1812)$. The P-LSM group was offered four one-on-one sessions annually with trained peer educators who provided individualized lifestyle modification advice. The C-LSM group was offered one annual one-on-one session with trained peer educators. Participants were followed up over a median of 3 years. The clinical study was given ethical approval from the Sri Lanka Medical Association Ethical Review Committee (ERC 07-010).

\subsection{Perspective}

The analysis took a broad health-care system perspective because in Sri Lanka the costs of diabetes prevention are borne by the public sector, but health-care treatment costs are met by a combination of both public sector payers and out-of-pocket private payers.

\subsection{Costs}

Within-trial resource use was recorded for each participant including the total number of sessions they attended, and blood tests administered for monitoring blood glucose levels. The cost of screening (travel costs, staff salaries, laboratory charges, office overheads) was estimated from trial records and apportioned across all subjects recruited to the study. The unit costs applied to each item of resource use in Sri Lankan Rupees (LKR) were inflated where necessary to 2017 prices (Table 1). The conversion rate for LKR 2017 to USD 2017 was 148 LKR to 1 USD (actual) [22] or 48 LKR to 1 USD (Purchasing Power Parity) [23]. The annual public sector cost of uncomplicated type 2 diabetes was based on 12 outpatient physician visits, and daily medication. On 
Table 1 Unit costs of treatment of diabetes and complications in Sri Lanka

\begin{tabular}{|c|c|c|}
\hline Item & Source & Unit cost (2017 LKR) \\
\hline \multicolumn{3}{|l|}{ Intervention costs } \\
\hline Cost per session & Hourly peer educator wage & LKR74 \\
\hline Screening cost & Trial screening costs/all subjects $(n=3539)$ & LKR7824 \\
\hline Blood sugar & Trial testing costs & LKR751 \\
\hline OGTT & Trial testing costs & LKR513 \\
\hline \multicolumn{3}{|c|}{ Diabetes-related medical costs } \\
\hline Outpatient visit & WHO Choice [32] & LKR434 \\
\hline Cardiovascular disease & Karan et al. 2014 [28] (India per year) & LKR $85,013^{\mathrm{b}}$ \\
\hline Nephropathy & Clarke et al. 2010 [29] (India per year) & LKR $44,243^{\mathrm{b}}$ \\
\hline Retinopathy & Rachapelle et al. 2013 [30] (India, societal) & LKR $14,290^{\mathrm{b}}$ \\
\hline Neuropathy & Clarke et al. 2010 [29] (India per year) & LKR36,769 \\
\hline \multicolumn{3}{|l|}{ Medications } \\
\hline Diabetes (metformin) & State Pharmaceuticals Corporation of Sri Lanka [33] & $\begin{array}{l}500 \mathrm{mg} 4 \times \text { a day } \\
\text { LKR1.00 per tablet }\end{array}$ \\
\hline
\end{tabular}

this basis, we estimated that the annual public sector cost of uncomplicated DM was LKR 6674.

In the absence of local data, direct medical costs of treating minor and major diabetic complications were taken from a recent systematic review of the economic costs of cardiovascular disease, diabetes, and diabetic complications in South Asia [24]. Direct medical costs of diabetic complications from this review were deemed appropriate for the Sri Lankan context given similarities in out-of-pocket expenditure between countries in the South Asia region considered in the review. The validity of the cost estimates was verified by local public health experts (authors DS, PS). All costs were inflated to 2017 prices.

\subsection{Outcomes}

We measured the effectiveness of LSM using disabilityadjusted life-years (DALYs) averted. The DALY is a generic measure of disease burden which was developed by the World Bank and the World Health Organization to inform health priority setting and health programme evaluation, particularly in low- and middle-income countries [25]. DALYs assign disability weights between 0 (no disability) and 1 (equivalent to death) to estimate the decline in health and functioning attributable to various illnesses and impairments. The health state disability weights used in the present study were taken from the Global Burden of Disease study (2016) [26]. These were uncomplicated diabetes mellitus (DM) (0.049); cardiovascular disease (0.179); chronic kidney disease due to DM (0.052); diabetic neuropathy (0.133); and severe vision impairment due to DM (0.184). DALYs were calculated by multiplying the relevant disability weight with the time between onset and follow up. It was assumed that DM onset occurred at the midpoint between a negative and positive test.

\subsection{Economic Model}

We constructed an economic model to predict costs and DALYs for a cohort of 1000 healthy individuals at risk of type 2 diabetes aged 22 years at presentation for the lifestyle modification intervention. Given the chronic and progressive nature of type 2 diabetes, a Markov model with a lifetime horizon was selected to capture downstream costs and consequences. The Markov model had nine health states including normal glucose tolerance (healthy), seven health states defined by complications from type 2 diabetes and death. The cohorts move through the Markov model in annual cycles accruing costs and DALYs depending on the health state and the group allocation. We assumed a legacy effect for lifestyle modification of 10 years [15]. The legacy effect and a 'do nothing' scenario as the control condition were tested in sensitivity analyses. Costs, outcome and transition probabilities were taken from the trial or other published estimates as needed. Costs and DALYs were discounted by $3 \%$ to account for time preferences. The model is specified in more detail in the electronic supplementary material (ESM). 


\subsection{Analysis}

The cost effectiveness of an intensive tri-monthly LSM intervention relative to an annual LSM intervention is presented as an incremental cost-effectiveness ratio (ICER), which is the difference in mean costs divided by the difference in mean DALYs, expressed as cost per DALY averted. Uncertainty around the point estimate was propagated using a scatterplot of 1000 bootstrapped differences in mean costs and mean DALYs. The impact on cost effectiveness according to age $(<18$ years, $\geq 18$ years) was also explored given a small but statistically significant lower fasting plasma glucose and 2-hour postOGTT (oral glucose tolerance test) plasma glucose level in the P-LSM as compared with the C-LSM group in participants aged $>18$ years of age only ( $p<0.05$ for both) reported in the main clinical paper [6]. The impact of uncertainty in the economic model was assessed through probabilistic sensitivity analysis, which used Monte Carlo methods to randomly vary all model parameters simultaneously. Cost effectiveness acceptability curves (CEACs) were derived from the joint distribution of the difference in costs and differences in DALYs averted. The CEAC shows the probability that that P-LSM is cost effective compared with C-LSM over willingness-to-pay thresholds of between 1 and 3 times the Sri Lankan GDP per capita, as per previous World Health Organization recommendations for low- and middle-income countries [27]. Analyses were conducted in STATA 15 and Excel.

\section{Results}

\subsection{Costs and Effects}

Resource use during the 3-year trial and associated mean costs per participant are summarized in Table 2. Over the 3 -year study period, the additional cost of the more intensive intervention was slightly offset by lower costs of type 2 diabetes complications. The mean difference in total costs was 188 LKR per participant (Bootstrap CI -107 to 484). Mean DALYs were 0.0013315 (SD 0.0108166) in the intensive intervention group and 0.0017329 (SD 0.0092226) in the less intensive control group. The difference in DALYs (or DALYs averted) was 0.0004 (Bootstrap CI -0.00019 to 0.00099). When stratified by age, the between-group differences in costs and DALYs averted were as follows: for participants $\geq 18$ years of age ( $n=1814),-6$ LKR (Bootstrap CI -273 to 262) and 0.0007 DALYs averted (Bootstrap CI -0.00016 to 0.00166 ); and for participants $<18$ years of age ( $n=1725$ ), 388 LKR (Bootstrap CI -159 to 935) and 0.000013 DALYs averted (Bootstrap CI -0.00097 to 0.00098). Note the very small difference in DALYs was due to the low incidence of type 2 diabetes and complications in a young pre-diabetic group observed in a 3-year RCT.

\subsection{Cost Effectiveness}

The difference in mean costs divided by the difference in mean effects yielded an ICER of 470,000 LKR per DALY averted over the 3-year trial period. Uncertainty around this point estimate is illustrated in Fig. 1. The scatterplots fall
Table 2 Mean cost per participant by item and group (SD) (2017 LKR)

\begin{tabular}{lllll}
\hline Item & $\begin{array}{l}\text { P-LSM }(n=1727) \\
\text { Mean (SD) }\end{array}$ & Notes & $\begin{array}{l}\text { C-LSM }(n=1812) \\
\text { Mean (SD) }\end{array}$ & Notes \\
\hline $\begin{array}{l}\text { A. Screening } \\
\text { Tests }\end{array}$ & $7824(\mathrm{nc})$ & & $7824(\mathrm{nc})$ & \\
B. FBS & $3113(817)$ & & $3205(775)$ & \\
C. OGTT & $2127(558)$ & & $2190(530)$ & \\
D. LSM counselling & $800(358)$ & 10.8 sessions $(4.8)$ & $309(80)$ & 4.2 sessions (1.1) \\
Intervention cost & $13,864(1675)$ & A+B+C+D & $13,528(1382)$ & A+B + C+D \\
DM-related costs & & & & \\
DM2 treatment & $145(864)$ & 59 cases & $199(1108)$ & 70 cases \\
CVD treatment & $99(4110)$ & 1 case & $0(0)$ & No cases \\
Nephropathy & $52(1512)$ & 2 cases & $234(3791)$ & 8 cases \\
Hypertension & $24(107)$ & 115 cases & $39(127)$ & 152 cases \\
Statin therapy & $10(73)$ & 41 cases & $12(85)$ & 46 cases \\
Total costs & $14,193(4749)$ & & $14,005(4148)$ & \\
\hline
\end{tabular}

$C$ - LSM low-intensity annual control LSM intervention, CVD cardiovascular disease, DM2 diabetes mellitus type 2, FBS fasting blood sugars, $L K R$ Sri Lankan Rupees, $L S M$ lifestyle modification, $n c$ not calculated, $O G T T$ oral glucose tolerance test, $P$-LSM intensive peer-educator-delivered tri-monthly LSM intervention, $S D$ standard deviation 
Fig. 1 Scatter plot of differences in costs in LKR and DALYs averted for P-LSM versus C-LSM over a 3-year trial period. $C$ - $L S M$ low-intensity annual control LSM intervention, $D A L Y s$ disability-adjusted life-years, $L K R$ Sri Lankan Rupees, $L S M$ lifestyle modification, $P$ - $L S M$ intensive peereducator-delivered tri-monthly LSM intervention

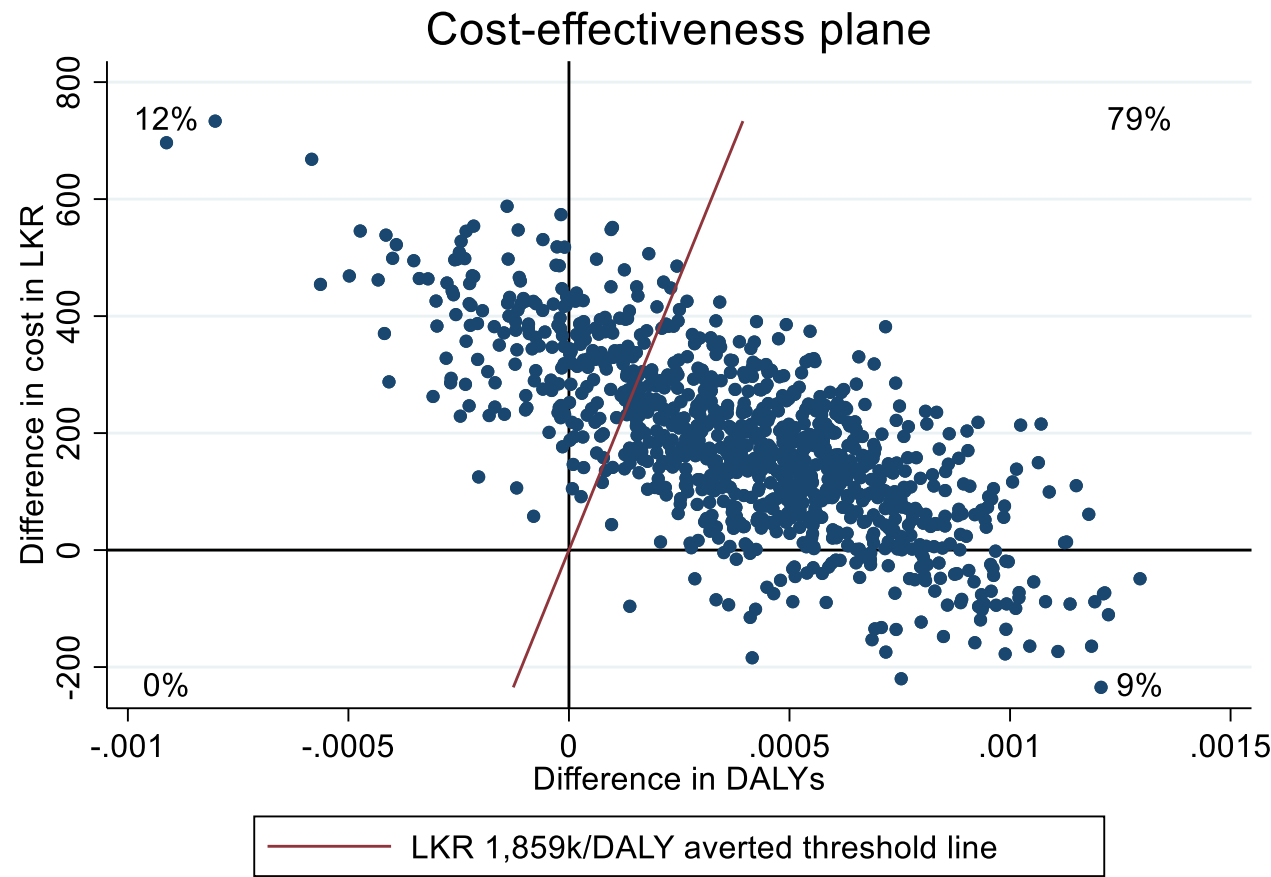

across three quadrants, most (79\%) fall in the upper righthand quadrant indicating that P-LSM was more effective than C-LSM but at an additional cost. Eight percent fall in the lower right-hand quadrant indicating that P-LSM was the dominant strategy improving outcomes at a lower cost, while $13 \%$ are in the upper left-hand quadrant indicating that P-LSM was dominated by C-LSM because it produces worse outcomes at an additional cost.

The threshold line represents a willingness-to-pay value of three times the Sri Lankan per capita GDP
(1,859,187 LKR per DALY averted). Seventy-eight percent of scatterplots fell below this threshold, indicating a $78 \%$ probability that P-LSM was cost effective compared with C-LSM. The results across a range of willingness-topay values based on the cost-effectiveness plane are plotted in Fig. 2, which shows the probability that P-LSM was cost effective compared with C-LSM. The probability that P-LSM was cost effective compared with C-LSM ranged from $57 \%$ at $1 \times$ GDP per capita, $72 \%$ at $2 \times$ GDP per capita to $78 \%$ at $3 \times \mathrm{GDP}$ per capita.
Fig. 2 Cost-effectiveness acceptability curve showing the probability that P-LSM is more cost effective than C-LSM over different values a decision maker is willing to pay in LKR to reduce DALYs. $C$ - $L S M$ lowintensity annual control LSM intervention, $D A L Y S$ disabilityadjusted life-years, $L K R$ Sri Lankan Rupees, $L S M$ lifestyle modification, $P$-LSM intensive peer-educator-delivered trimonthly LSM intervention

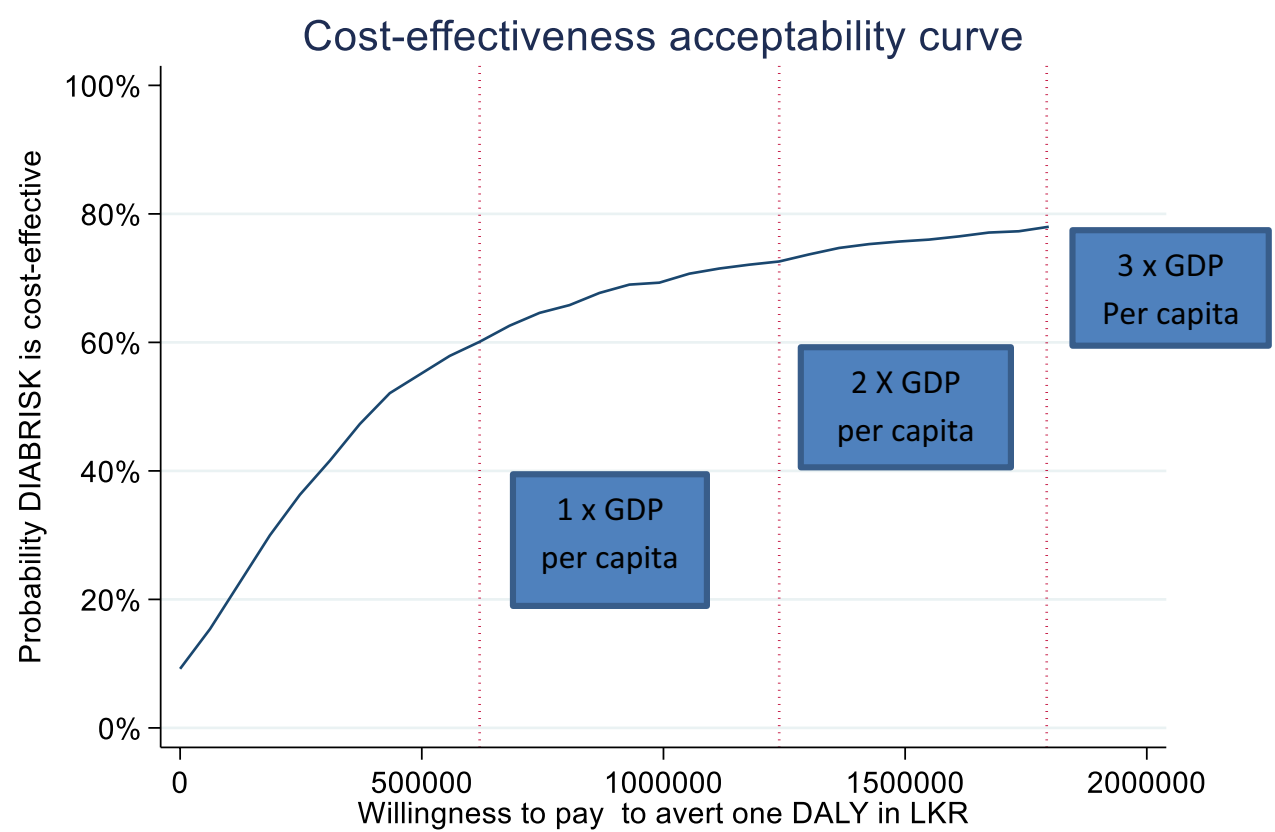


Cost effectiveness varied according to age. In participants aged $\geq 18$ years, P-LSM dominated C-LSM, averting more DALYs at a lower cost, and was clearly more cost effective with the probability of cost effectiveness ranging from 89 to $93 \%$. In participants younger than 18 years, the cost per DALY averted was 29,612,403 LKR with the probability of cost effectiveness ranging from $27 \%$ to $43 \%$.

\subsection{Economic Model}

The economic model predicted that P-LSM would dominate C-LSM with lifetime savings of 6000 LKR and averting an additional 0.456 DALYs per participant. The probability that P-LSM was cost effective compared with C-LSM ranged from $84 \%$ at $1 \times$ GDP per capita, $85 \%$ at $2 \times$ GDP per capita to $85 \%$ at $3 \times$ GDP per capita. The detailed results from the model appear in the ESM. Sensitivity analysis was used to explore two assumptions in the economic model. Under the 'do nothing' scenario, where participants in the control group incurred zero intervention costs, P-LSM continued to dominate C-LSM, saving 4836 LKR and averting 0.456 DALYs over participant lifetimes. When the 10-year legacy effect assumption was replaced by an assumption of no effect beyond the trial period, P-LSM continued to dominate C-LSM, saving 1949 LKR and averting 0.149 DALYs over the lifetime of participants.

\section{Discussion}

This economic analysis found that an intensive peer-educator-delivered lifestyle modification programme for healthy young Sri Lankans at risk of type 2 diabetes was cost effective from the Sri Lankan health-care system perspective. The within-trial comparison of intervention costs, type 2 diabetes-related health care costs, and DALYs averted showed that P-LSM was more cost effective than C-LSM with a probability of cost effectiveness between 57 and $78 \%$ based on WHO health investment guidelines for LMICs. The low cost of the peer-delivered intervention was partially offset by savings from a relatively lower incidence of type 2 diabetes and type 2 diabetes complications over the 3 -year trial period.

In long-term conditions such as type 2 diabetes, most of the savings and benefits from effective interventions will occur well after the time horizon of clinical trials. This is particularly true for preventative interventions aimed at young people at risk from type 2 diabetes. This is reflected in our within-trial analysis, which did not find evidence for cost effectiveness in pre-diabetic participants $<18$ years old due to the low observed incidence of type 2 diabetes and complications and associated costs and DALYs. In contrast, the intervention was clearly cost effective for young adults aged 18 years or older where the intervention costs were entirely offset by health care savings from avoided disease. When the different risks observed in the trial for type 2 diabetes, type 2 diabetes complications and pre-diabetes type 2 diabetes were modelled together with risks from the literature, P-LSM saved the Sri Lankan health care system money due to lower costs from type 2 diabetes and type 2 diabetes complications over the young people's lifetimes. In the long run, P-LSM was predicted to be more cost effective than C-LSM with a probability of cost effectiveness between 84 and $85 \%$ using WHO health investment guidelines for LMICs. The lifetime results from the model were robust to changes in underlying assumptions about costs and effects.

Our results are consistent with findings from other published economic evaluations that have found lifestyle modification to be cost effective overall [28]. The only study to date that that has considered intensity of lifestyle modification agreed with our finding that more intensive interventions were cost effective relative to low-intensity programmes [14]. Our findings are also consistent with other economic evaluations of lifestyle modification interventions for type 2 diabetes in LMIC settings. Liu and colleagues used economic modelling to demonstrate that group-based lifestyle modification interventions in a representative sample of Chinese people aged $\geq 25$ years resulted in cost savings at all initiation ages, but especially in a younger cohort aged 25 years (17). The Indian Diabetes Prevention programme reported cost effectiveness as evaluated by the amount spent to prevent one case of diabetes within the 3-year trial period and demonstrated that lifestyle modification and metformin were cost-effective interventions for preventing diabetes among individuals aged 35-55 years with impaired glucose tolerance in South India [29].

The strength of this evaluation lay in the use of intervention resource use and effectiveness data from the Sri Lankan context collected through the DIABRISK-SL trial. Furthermore, the study considered costs and consequences for a younger at-risk population that are lacking in the existing literature and are relevant particularly for South Asian populations who are predisposed to early onset of type 2 diabetes. Most existing cost-effectiveness studies consider pre-diabetic populations who have higher risk of progressing to type 2 diabetes; however, from a prevention perspective it is important to consider at-risk populations who lie below the pre-diabetic threshold. This evaluation contributes toward addressing this gap by considering a population with normal glucose tolerance at baseline.

The results from this economic evaluation are, however, subject to limitations. The lack of unit cost data for diabetic complications for the Sri Lankan context meant that we relied on data from a systematic review of costs from other South Asian countries. We were unable to apply discounting to the within-trial data due to uncertainties around timing 
of service use and the incidence of type 2 diabetes and type 2 diabetes complications. However, discounting was implemented in the model where time preferences for lifetime costs and benefits would arguably be more relevant. We were unable to include overhead costs as these could not be disentangled from the costs of research and the pre-existing public screening programme established in Sri Lanka.

We used the WHO recommendation of 1-3 $\times$ GDP to interpret our cost per DALY results; however, this guidance was withdrawn due to perceived shortcomings including affordability concerns and instead country-specific thresholds in LMICs were recommended [30]. Despite this, almost no LMICs, including Sri Lanka, have published explicit cost-effectiveness thresholds to assess whether the additional costs are worth the additional health gains from new health care interventions in their national contexts [31]. Readers can apply their own thresholds to interpret the CEACs presented in Fig. 2 and Fig. S4 (see ESM) either in terms of Sri Lankan rupees or Sri Lankan GDP multiples.

\section{Conclusions}

Efforts to reduce the global health and economic burden of diabetes should emphasize prevention of diabetes, or delaying its onset, through enhancing health behaviours and diets at the population level, and early detection and management of high-risk individuals. This economic evaluation adds to the growing literature on the cost effectiveness of lifestyle modification with much-needed data on costs and effects of implementing such programmes in a low-income setting. Policy makers in LMICs with limited health budgets such as Sri Lanka face important choices in designing large-scale prevention programmes-whether to screen for diabetes risk or target known at-risk individuals with pre-diabetes; whether to use community or lay health workers to deliver such interventions; the duration of interventions; and the intensity with which they are delivered. This economic evaluation indicates that lifestyle interventions delivered at low intensity may minimize budget impact in the short term, but more intensive lifestyle modification interventions delivered at younger ages will maximize reductions in diabetes incidence and costs in the long term and are therefore better value for money.

Supplementary Information The online version contains supplementary material available at https://doi.org/10.1007/s41669-021-00284-5.

\section{Declarations}

Funding No funding was received to conduct this economic analysis. The DAIBRISK-SL project was supported by a BRIDGES Grant from the International Diabetes Federation and a grant awarded by the Diabetes Association of Sri Lanka. BRIDGES, an International Diabetes
Federation project, is supported by an educational grant from Lilly Diabetes.

Conflict of interest The authors declare they have no conflicts of interest.

Ethics All participants provided written informed consent and the study was given ethical approval from the Sri Lanka Medical Association Ethical Review Committee (ERC 07-010). Permission from the Ministry of Education was obtained for this study, which was conducted under the Good Clinical Practice Guidelines and according to the principles expressed in the Declaration of Helsinki for clinical research.

Consent to participate All participants provided informed, written consent for participation in the study.

Consent for publication All participants provided informed, written consent prior to participating in the study, which included consent for de-identified data to be used in future publications.

Availability of data and materials The de-identified datasets generated during the current study will be considered for sharing by the corresponding author upon reasonable request.

Code availability The code generated during the current study will be considered for sharing by the corresponding author upon reasonable request.

Author contributions JS wrote the manuscript and analysed the data; MK contributed to the manuscript and analysed the economic model; AM researched data; DS reviewed/edited the manuscript; PS reviewed/ edited the manuscript; MW reviewed/edited the manuscript and contributed to the manuscript and JK reviewed/edited manuscript and contributed to the manuscript.

Open Access This article is licensed under a Creative Commons Attribution-NonCommercial 4.0 International License, which permits any non-commercial use, sharing, adaptation, distribution and reproduction in any medium or format, as long as you give appropriate credit to the original author(s) and the source, provide a link to the Creative Commons licence, and indicate if changes were made. The images or other third party material in this article are included in the article's Creative Commons licence, unless indicated otherwise in a credit line to the material. If material is not included in the article's Creative Commons licence and your intended use is not permitted by statutory regulation or exceeds the permitted use, you will need to obtain permission directly from the copyright holder. To view a copy of this licence, visit http://creativecommons.org/licenses/by-nc/4.0/.

\section{References}

1. International Diabetes Federation. International Diabetes Federation. Diabetes Atlas 2017. 2017. http://diabetesatlas.org/IDF_ Diabetes_Atlas_8e_interactive_EN/.

2. Seuring T, Archangelidi O, Suhrcke M. The economic costs of type 2 diabetes: a global systematic review. Pharmacoeconomics. 2015;33(8):811-31.

3. Nanditha A, Ma R, Ramachandran A, Snehalatha C, Chan J, Chia $K$. Diabetes in Asia and the Pacific: implications for the global epidemic. Diabetes Care. 2016;39(3):472-85. 
4. Ramachandran A, Ma R, Snehalatha C. Diabetes in Asia. Lancet. 2010;375(9712):408-18.

5. Hu F. Globalization of diabetes: the role of diet, lifestyle, and genes. Diabetes Care. 2011;34(6):1249-57.

6. Wijesuriya M, Fountoulakis N, Guess N, Banneheka S, Vasantharajah L, Gulliford M, et al. A pragmatic lifestyle modification programme reduces the incidence of predictors of cardiometabolic disease and dysglycaemia in a young healthy urban South Asian population: a randomised controlled trial. BMC Med. 2017;30(1):146.

7. Bommer C, Heesemann E, Sagalova V, Manne-Goehler J, Atun $\mathrm{R}$, Bärnighausen $\mathrm{T}$. The global economic burden of diabetes in adults aged 20-79 years: a cost-of-illness study. Lancet Diabetes Endocrinol. 2017;15(6):423-30.

8. NCD Risk Factor Collaboration. Worldwide trends in diabetes since 1980: a pooled analysis of 751 population-based studies with 4.4 million participants. Lancet. 2016;387(10027):1513-30.

9. Li G, Zhang P, Wang J, Gregg E, Yang W, Gong Q. The long-term effect of lifestyle interventions to prevent diabetes in the China Da Qing Diabetes Prevention Study: a 20-year follow-up study. Lancet. 2008;371(9626):1783-9.

10. Lindström J, Peltonen M, Eriksson J, Ilanne-Parikka P, Aunola S, Keinänen-Kiukaanniemi S. Improved lifestyle and decreased diabetes risk over 13 years: long-term follow-up of the randomised Finnish Diabetes Prevention Study (DPS). Diabetologia. 2013;56(2):284-93.

11. Diabetes Prevention Program Research Group, Knowler W, Fowler S, Hamman R, Christophi C, Hoffman H. 10-year follow-up of diabetes incidence and weight loss in the Diabetes Prevention Program Outcomes Study. Lancet. 2009;374(9702):1677-86.

12. Ramachandran A, Snehalatha C, Mary S, Mukesh B, Bhaskar A, Vijay V. The Indian Diabetes Prevention Programme shows that lifestyle modification and metformin prevent type 2 diabetes in Asian Indian subjects with impaired glucose tolerance (IDPP-1). Diabetologia. 2006;49(2):289-97.

13. Kosaka K, Noda M, Kuzuya T. Prevention of type 2 diabetes by lifestyle intervention: a Japanese trial in IGT males. Diabetes Res Clin Pract. 2005;67(2):152-62.

14. Roberts S, Craig D, Adler A, McPherson K, Greenhalgh TBM. Economic evaluation of type 2 diabetes prevention programmes: Markov model of low- and high-intensity lifestyle programmes and metformin in participants with different categories of intermediate hyperglycaemia. BMC Med. 2018;30(1):16.

15. Neumann A, Schwarz P, Lindholm L. Estimating the cost-effectiveness of lifestyle intervention programmes to prevent diabetes based on an example from Germany: Markov modelling. Cost Eff Resour Alloc. 2011;9(1):1-3.

16. van Wier M, Lakerveld J, Bot S, Chinapaw M, Nijpels G, van Tulder M. Economic evaluation of a lifestyle intervention in primary care to prevent type 2 diabetes mellitus and cardiovascular diseases: a randomized controlled trial. BMC Fam Pract. 2013;14(100967792):45.

17. Liu X, Li C, Gong H, Cui Z, Fan L, Yu W. An economic evaluation for prevention of diabetes mellitus in a developing country: a modelling study. BMC Public Health. 2013;13(729):1-11.
18. Irvine L, Barton G, Gasper A, Murray N, Clark A, Scarpello T. Cost-effectiveness of a lifestyle intervention in preventing type 2 diabetes. Int J Technol Assess Health Care. 2011;27(4):275-82.

19. Gillies CL, Lambert PC, Abrams KR, Sutton AJ, Cooper NJ, Hsu RT. Different strategies for screening and prevention of type 2 diabetes in adults: cost effectiveness analysis. BMJ. 2008;336(7654):1180-4.

20. Neumann A, Lindholm L, Norberg M, Schoffer O, Klug SJ, Norstrom F. The cost-effectiveness of interventions targeting lifestyle change for the prevention of diabetes in a Swedish primary care and community based preventio (Placeholder1)n program. Eur J Health Econ. 2017;18(7):905-19.

21. Palmer AJ, Tucker DMD. Cost and clinical implications of diabetes prevention in an Australian setting: A long-term modeling analysis. Prim Care Diabetes. 2012;6(2):109-21.

22. LKR:USD 2017. https://www.poundsterlinglive.com/best-excha nge-rates/us-dollar-to-sri-lankan-rupee-exchange-rate-on-201705-17. Accessed 08 June 2021.

23. LKR:USD PPP. 2017. https://www.quandl.com/data/ODA/LKA_ PPPEX-Sri-Lanka-Implied-PPP-Conversion-Rate-LCU-per-USD. Accessed 08 June 2021.

24. Walker I, Garber F, Wright J, Newell I, Athiraman N, Khan NS, et al. The economic costs of cardiovascular disease, diabetes mellitus, and associated complications in South Asia: a systematic review. Value Health Reg Issues. 2018;15C:12-26.

25. Chen A, Jacobsen K, Deshmukh A, Cantor S. The evolution of the disability-adjusted life year (DALY). Socioecon Plann Sci. 2015;49:10-5.

26. Global Health Data Exchange. Global Burden of Disease Study Seattle: Institute for Health Metrics and Evaluation; 2016. http:// ghdx.healthdata.org/gbd-2016.

27. Commission on Macroeconomics and Health. Macroeconomics and Health: Investing in Health for Economic Development. Geneva: World Health Organisation; 2001.

28. Roberts S, Barry E, Craig D, Airoldi M, Bevan G, Greenhalgh T. Preventing type 2 diabetes: Systematic review of studies of cost-effectiveness of lifestyle programmes and metformin, with and without screening, for pre-diabetes BMJ Open. 2017;7(11):e017184.

29. Ramachandran A, Snehalatha C, Yamuna A, Mary S, Ping Z. Costeffectiveness of the interventions in the primary prevention of diabetes among Asian Indians. Diabetes Care. 2007;30(10):2548-52.

30. Bertram M, Lauer J, De Joncheere K, Edejer T, Hutubessy R, Kienya M, et al. Cost-effectiveness thresholds: pros and cons. Bull World Health Organ. 2016;94:925-30.

31. Cameron D, Ubels J, Norström F. On what basis are medical cost-effectiveness thresholds set? Clashing opinions and an absence of data: a systematic review. Glob Health Action. 2018;11(1):1447828.

32. WHO Choice. https://www.who.int/choice/cost-effectiveness/ inputs/country_inpatient_outpatient_2010.pdf?ua=1. Accessed 19 Dec 2019.

33. State Pharmaceuticals Corporation of Sri Lanka. www.spc.lk. Accessed 17 Apr 2019 